\title{
Correction to: Container Handling in Automated Yard Blocks
}

\section{Correction to:}

F. Covic et al., Container Handling in Automated Yard Blocks, Contributions to Management Science, https://doi.org/10.1007/978-3-030-05291-1

The book was incorrectly published with the wrong footnotes for chapters 4,7 and 8 . This has been updated in this book.

\footnotetext{
The updated online versions of the chapters can be found at https://doi.org/10.1007/978-3-030-05291-1_4 https://doi.org/10.1007/978-3-030-05291-1_7 https://doi.org/10.1007/978-3-030-05291-1_8 